\title{
Tracing the horizontal transport of microplastics on rough surfaces
}

\author{
Hannes Laermanns ${ }^{1}$, Moritz Lehmann² ${ }^{2}$ Marcel Klee ${ }^{1}$, Martin G. J. Löder ${ }^{3}$, Stephan Gekle ${ }^{2}$ and Christina \\ Bogner $^{1 *}$ (D)
}

\begin{abstract}
Occurrence and distribution of microplastics in different ecosystems have recently become subjects of numerous studies. However, to date the research has focused mainly on marine and freshwater ecosystems and widely neglected terrestrial environments. Only recently, first studies investigated the microplastics contamination of soils. Therefore, we know little about the transport mechanisms of microplastics in soils and sediments and virtually nothing about their surface transport. In this study we investigate surface transport mechanisms by tracking fluorescent, irregularly shaped polymethyl methacrylate (PMMA) particles in real time in a laboratory setup. In 108 experimental runs, we vary the irrigation rates, inclinations and surface roughnesses. Additionally, we simulate the small-scale flow patterns to resolve the role of the roughness-induced microrelief. Our results suggest that microplastics are transported along preferential pathways resulting from the micro- and macrorelief, which can be correlated to the flow pattern observed in the computer simulation. Our model study facilitates a deeper insight into microplastic transport on different soil surfaces and serves as a pilot for investigating mechanisms of horizontal microplastic transport. However, microplastics are a diverse group of contaminants with varying shapes, densities and sizes. Therefore, for a full understanding of transport of microplastics in terrestrial environments, it is important to address these properties as well as more variable surfaces for horizontal migration and to include vertical transport mechanisms in future research.
\end{abstract}

Keywords: Microplastics, Surface transport, Surface roughness, PMMA, sCMOS camera, Particle tracking

\section{Introduction}

The large-scale production of synthetic polymers started in the 1950s and over the last seven decades the amount of produced plastic has exceeded eight billion tons [1]. In 2019, plastic production reached 57.9 million tons in Europe and 368 million tons globally [2]. Plastic products are durable and resist to degradation [1], however, the accompanying environmental challenges caused by the increasing amount of plastic waste entering the environment have been neglected for a long time. Due to its poor biodegradability, plastic waste could remain in the environment for centuries leading to an accumulation

\footnotetext{
*Correspondence: christina.bogner@uni-koeln.de

${ }^{1}$ Ecosystem Research Group, Institute of Geography, Faculty of Mathematics and Natural Sciences, University of Cologne, Albertus Magnus Platz, 50923 Cologne, Germany

Full list of author information is available at the end of the article
}

and a potential threat to our ecosystems $[1,3]$. While for a long time only large items of plastic waste have been studied, microplastics came more into focus during the last decade [3]. Microplastics are in general defined as particles smaller than $5 \mathrm{~mm}$ [4] and occur as primary and secondary microplastics. Primary microplastics are intentionally produced micro particles like pellets and beads. Secondary microplastics originate from abrasion of plastic products or fragmentation of macroplastics by a combination of e.g. elevated temperature, light, exposure to water, friction from wind and water or exposure to organisms, resulting in thermo-oxidative degradation, photo-oxidation, hydrolysis, mechanical breakdown and biodegradation $[1,5,6]$. Due to their manifold origins and degradation pathways, microplastics are a diverse group of contaminants with a broad range of shapes, densities, sizes, polymers and additives [7]. It is best to think about
Springer Open

(c) The Author(s). 2021 Open Access This article is licensed under a Creative Commons Attribution 4.0 International License, which permits use, sharing, adaptation, distribution and reproduction in any medium or format, as long as you give appropriate credit to the original author(s) and the source, provide a link to the Creative Commons licence, and indicate if changes were made. The images or other third party material in this article are included in the article's Creative Commons licence, unless indicated otherwise in a credit line to the material. If material is not included in the article's Creative Commons licence and your intended use is not permitted by statutory regulation or exceeds the permitted use, you will need to obtain permission directly from the copyright holder. To view a copy of this licence, visit http://creativecommons.org/licenses/by/4.0/. 
their properties as a continuum rather than in discrete categories [8]. Today microplastics are found in every environmental compartment and region of the globe from megacities to even the remotest arctic areas [9-11].

While the contamination of oceans with microplastics and their transport in the marine environment have become a widely considered research field, our knowledge on the distribution and transport of microplastics in terrestrial environments is still limited [10, 12-14]. This is partly due to the analytical challenges because the extraction of microplastics from mineral and organicrich soils and sediments is much more complicated than from aquatic samples [15-17]. Additionally, we still lack a standard analytical protocol which is a prerequisite for a proper comparison of data from different studies $[18,19]$. Furthermore, the distribution of microplastics in the terrestrial environments depends on diverse sources and transport pathways. Sources can be manifold and include e.g. the introduction of microplastics via sewage sludge, compost application or plastic mulching in agriculture, littering or waste mismanagement and the fragmentation and abrasion of plastic particles from plastic products with outdoor use $[17,20,21]$. Once in the environment, microplastics may be transported by e.g. wind and water, and may finally be deposited and potentially accumulate in different environmental compartments [20, 22, 23]. Indeed, terrestrial soils are suggested as a large sink for microplastics [10, 24, 25].

While first experimental evidence on factors affecting vertical transport processes of microplastics in soils and sediments like e.g. particle size or presence of preferential flow paths are published [26-30], studies on horizontal transport are lacking to date [20,31]. To fill this gap, tracking microplastics during their transport could generate the necessary data and help to understand the relevant transport parameters and mechanisms. Particle tracking, also referred to as particle tracing [32], has a long history in sedimentology [33]. In this discipline it is understood as the analysis of the spatial and temporal distribution of single natural or artificial components of the sediment that are marked and observed during transport [32]. In environmental sciences, sand particles are tracked for example along fluvial trajectories [34], along coasts [35] or on tidal inlets [36]. Furthermore, particles can also be tracked under laboratory condition on a far smaller scale including vertical and horizontal transport pathways with a high temporal and spatial resolution [37-40].

Nowadays specific marking or tagging the particles of interest with an identifiable signature largely facilitates the tracking [32]. Since the use of radioactivity has shown its limitations and is seen critically due to environmental and health concerns, fluorescent dyes are commonly used $[32,41]$. Therefore, rhodamine or anthracene serve frequently as fluorescent coatings in combination with addi-

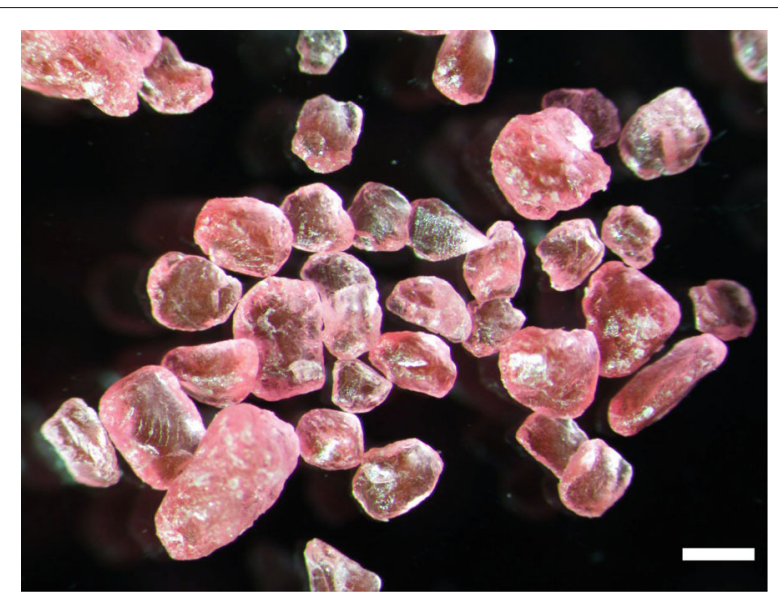

Fig. 1 A subsample of PMMA particles used in the experiments. The figure was cropped, reduced in size and the scale bar enlarged for better visibility in ImageJ [45]. The scale is $1 \mathrm{~mm}$ large

tive binding agents for particle tracking [32, 42]. Advances in real-time imaging and image processing allow to trace temporal and spacial distribution of particles, at least under laboratory conditions. Hardy and colleagues [40], for example, developed a real-time approach to trace the movement of sand particles with fluorescent coating during rainfall events by using fluorescent videography techniques. They could visualise and measure transport parameters like direction and travel distance of sand particles. However, most of the studies dealing with particle tracing focus on the spatial distribution as a result of transport processes. Real-time tracking of (natural) particles as demonstrated by Hardy and colleagues in a laboratory and field setup for terrestrial environments remains an exception [40, 43]. Nizzetto and colleagues [31] suggested in their theoretical study that microplastics could be transported similarly to natural particles. However, data to confirm this statement and to parametrize models is lacking [44]. Nevertheless, such data are of utmost importance for a mechanistic understanding of transport processes of microplastics in the environment.

In this study, we constructed a laboratory setup for realtime particle tracing and observed fluorescent microplastics in irrigation experiments. We analysed the influence of different irrigation rates, inclinations and surface roughnesses on the movement of irregularly shaped polymethyl methacrylate microplastics.

\section{Material and methods}

\section{Microplastic particles}

We used UV-fluorescence-labelled polymethyl methacrylate (PMMA) particles. PMMA, also known as acrylic glass, has a density of 1.18 to $1.19 \mathrm{~g} \mathrm{~cm}^{-3}$ and is thus heavier than water. The source material was purchased already fluorescence-labelled from LLV-shop (https://www.llv- 


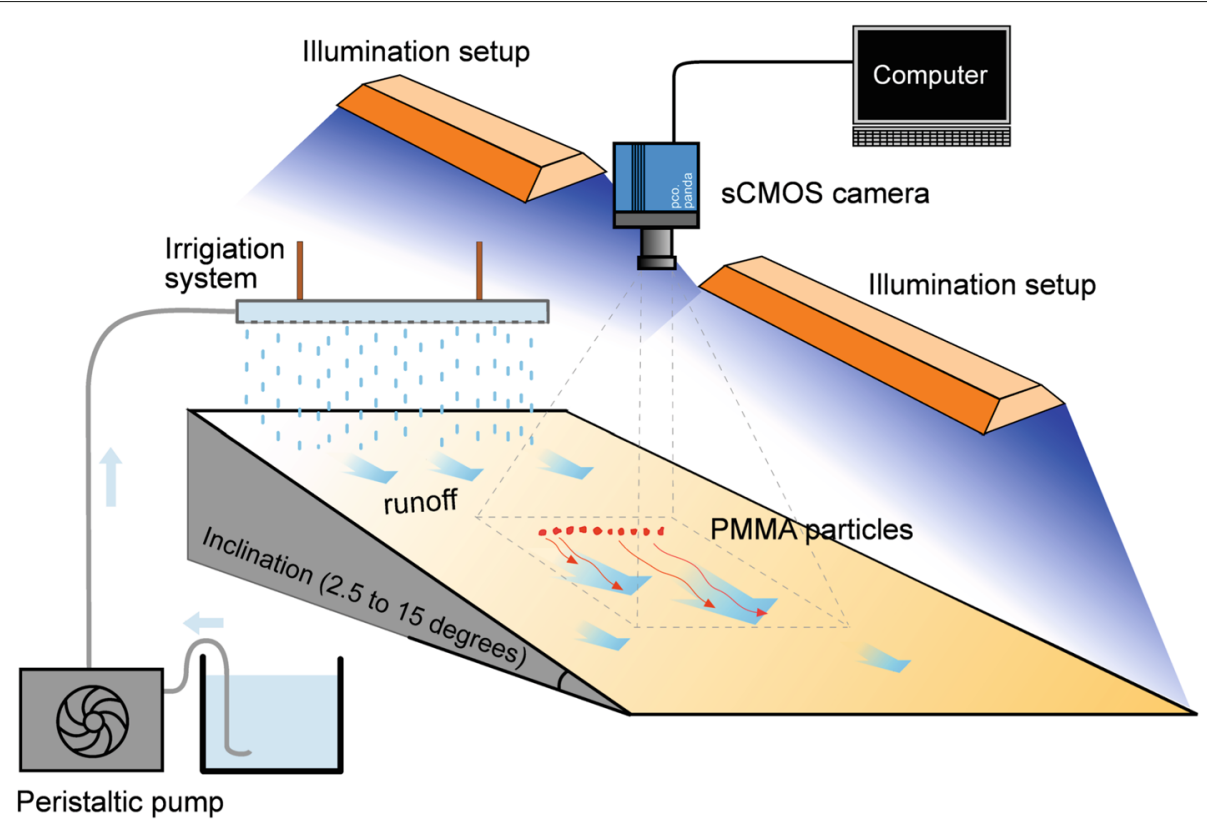

Fig. 2 Laboratory setup with the irrigation and illumination systems. The PMMA particles are positioned in row vertically below the camera

shop.de) in form of raw pellets (Art. Nr. M 13344). Subsequently, microplastic particles in the desired size range were produce by cryo-milling and sieving. We chose PMMA because among the nonfloating polymers, it has a medium density. Additionally, it can be milled relatively easily in contrast to other polymers (glass transition temperature above $100{ }^{\circ} \mathrm{C}$ ). We determined the size range on a subsample by taking pictures with a stereomicroscope (Leica M50, Leica Microsystems \& Olympus DP 26 camera, Olympus Corporation) and the imaging software cellSens (Olympus Corporation). The maximum length and width of 200 particles were measured in the free software ImageJ [45]. The particles were irregularly shaped with a mean particle length of $1215 \pm 227 \mu \mathrm{m}$ ( \pm standard deviation), a mean particle width of $919 \pm 186 \mu \mathrm{m}$ and a mean length-to-width ratio of 1.32 (Fig. 1). The smallest recorded length and width were $842 \mu \mathrm{m}$ and $485 \mu \mathrm{m}$, respectively, the largest recorded length and width were $2456 \mu \mathrm{m}$ and $1648 \mu \mathrm{m}$, respectively. The sizes of particles used during the experiments were estimated via image analysis in Python (c.f. "Image analysis" section).

\section{Experimental setup}

The experimental setup consisted of an inclined rough surface of $545 \times 400 \mathrm{~mm}$ that was irrigated at its upper edge (Fig. 2). To limit all particle movements to surface transport and avoid particle loss by infiltration, we affixed a sand surface with tile glue (Probau GmbH, Cologne, Germany) on two wooden chipboards. For one board we used medium sand $(<630 \mu \mathrm{m}$, mean grain size $408 \mu \mathrm{m})$, for the other coarse sand $(630-2000 \mu \mathrm{m}$, mean grain size $1,052 \mu \mathrm{m})$ to create two different levels of roughness (in the following referred to as 'fine surface' and 'coarse surface', respectively). For each experimental run ten PMMA particles (once 9 and twice 11) were placed in a row spaced by roughly one $\mathrm{cm}$ in the center of the surface. To generate runoff on the surface, an irrigation system was built using an ISMATEC ISM930C-IPC04 peristaltic pump (Cole-Parmer GmbH, Wertheim, Germany) equipped with four channels. The irrigation was placed at the upper edge of the surface to avoid any splash effects at the center where the PMMA particles were located. Therefore, the artificial rain did not hit the microplastics directly, but generated a runoff that flowed down the plate (Supplementary Figures S1 and S2). We irrigated the surface with 4.8, 7.2 or 10.44 $\mathrm{L} \mathrm{h}^{-1}$. During the experiments, the surface was inclined by $2.5,5,7.5,10,12.5$ and $15^{\circ}$. Altogether 36 different combinations of surface roughness (2), irrigation rates (3) and inclinations (6) were tested in three repetitions amounting to 108 runs in total of 50 seconds each.

\section{Camera and illumination setup}

During the experiments, we took pictures with an sCMOS (advanced scientific complementary metaloxide-semiconductor) high resolution pco.panda $4.2 \mathrm{cam}$ era (PCO AG, Kehlheim, Germany) equipped with a monochrome sensor with $2048 \times 2048$ pixels of $6.5 \times$ 


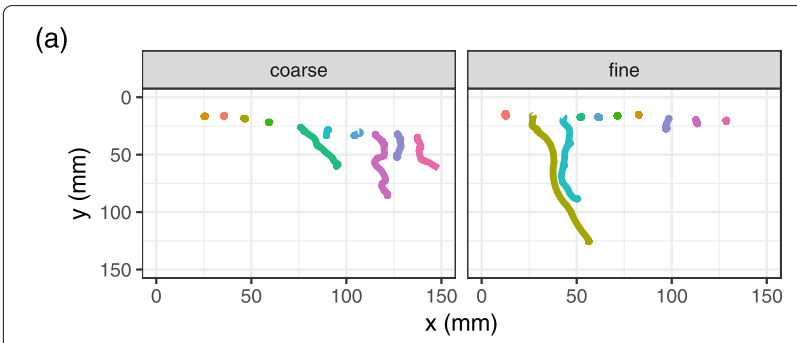

(b)

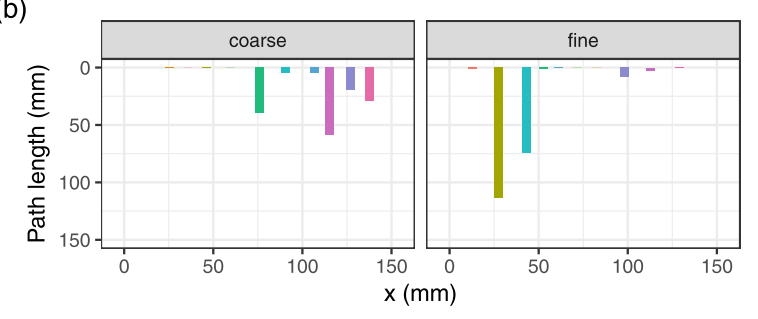

$$
\begin{array}{r}
\text { Particle ID }-0-2-4-6-8 \\
-1-3-7-9
\end{array}
$$

Fig. 3 Examples of (a) trajectories of particles and (b) corresponding path lengths. These are results from one of the runs on the coarse surface with inclination $2.5^{\circ}$ and an irrigation rate of $10.44 \mathrm{~L} \mathrm{~h}^{-1}$ and from a run with the same parameters but on the fine surface. Particle ID corresponds to an automatic numbering of PMMA articles sorted by the $y$ coordinate

$6.5 \mu^{2}$ and a Ricoh FL-BC2518-9M $25 \mathrm{~mm}$ objective. An sCMOS sensor has the advantage to combine a large field-of-view, low electrical noise and a high frame rate compared to a CCD (charge-coupled device) sensor [46]. The exposure time of the camera was set to $100 \mathrm{~ms}$, resulting in a frame rate of 10 frames per second and
500 pictures during the 50 seconds of recording for each experimental run. The camera was positioned $30 \mathrm{~cm}$ vertically above the PMMA particles in the central part of the surface and recorded an area of $150 \times 150 \mathrm{~mm}$ (Supplementary Figure S2). The camera was equipped with an EFFO-FLR-BN630-M40.5 optical filter (Midwest Optical Systems Inc., Palatine, Illinois, United States) to limit the recorded spectrum to a narrow spectral range around $630 \mathrm{~nm}$, which corresponds to the fluorescence of the PMMA particles. Two EFFI-FLEX-15-465-SD-P2 LED bars (EFFILUX GmbH, Les Ulis, France) with an emission wave length of $465 \mathrm{~nm}$ (blue light) were fixed laterally above the rough surface to excite the fluorescence of the PMMA particles. Natural illumination was minimized by darkening the laboratory to reduce noise in the pictures.

\section{Image analysis}

We analysed the images using Python 3.8 (Python Software Foundation, https://www.python.org/) and R 4.0.3 [47]. Some of the images contained artefacts due to residual ambient light which resulted in blurred particles' edges. Additionally, some particles moved abruptly and their trajectories appeared as lines in the images (Supplementary Figure S4). To remove the artefacts, we first preprocessed the images by calculating the centre of mass of every particle and then simplified them to equally sized circles centered around their centres of mass. This step increased the accuracy of particle tracking. The latter was done with TrackPy version 0.4.2 [48], a Python package that calculates particle trajectories or paths. Sub-

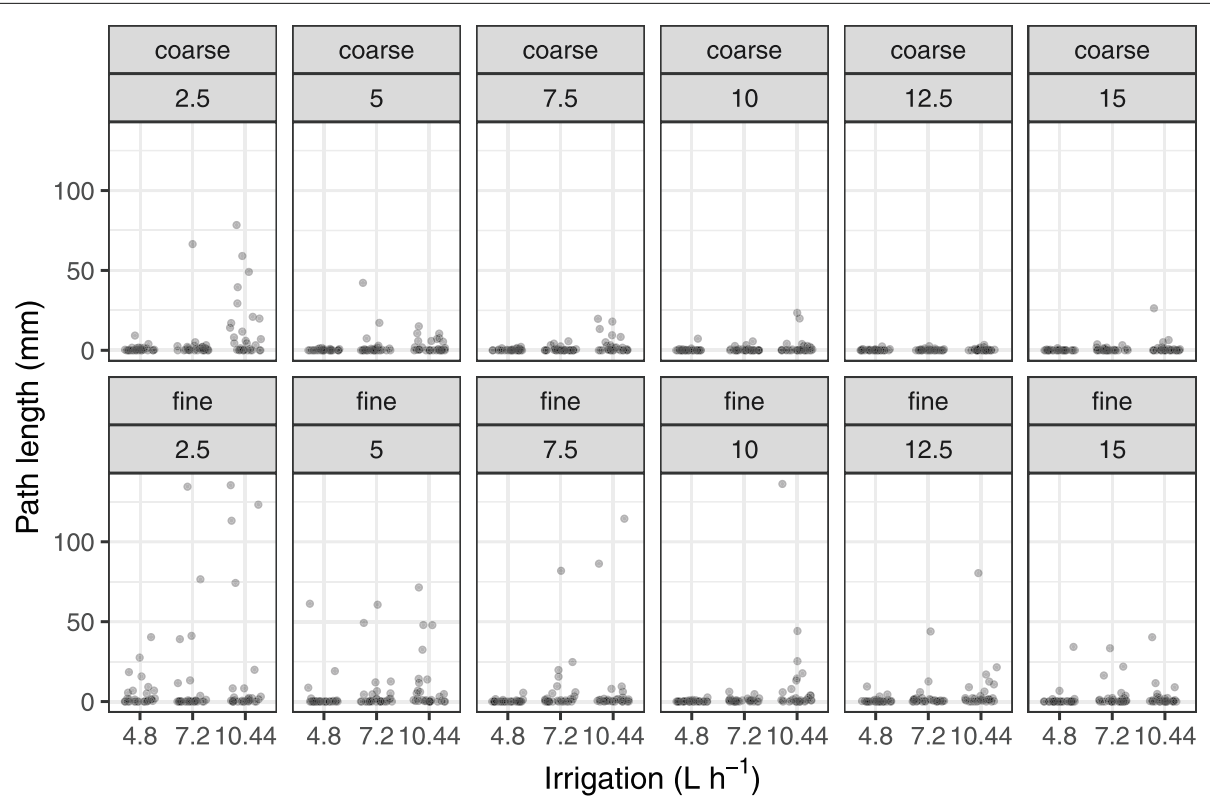

Fig. 4 Variation of path lengths with increasing irrigation and inclination on the coarse and the fine surfaces 
Table 1 Estimated parameters of the linear model with confidence intervals shown in parentheses

\begin{tabular}{ll}
\hline Model parameter & Estimate (Cl) \\
\hline Intercept $(\mathrm{mm})$ & $1.76(1.2,2.3)$ \\
Equivalent diameter $\left(\mathrm{mm} \mathrm{\mu m}^{-1}\right)$ & $-2.96 \mathrm{E}-03(-4.49 \mathrm{E}-03,-1.54 \mathrm{E}-03)$ \\
Inclination $\left(\mathrm{mm} \mathrm{degree}^{-1}\right)$ & $-0.46(-0.69,-0.27)$ \\
Irrigation $\left(\mathrm{mm} \mathrm{L}^{-1} \mathrm{~h}\right)$ & $0.89(0.57,1.3)$ \\
Roughness fine $(\mathrm{mm})$ & $3.33(1.97,5.11)$ \\
\hline
\end{tabular}

The parameters correspond to $\beta_{i}$ in Eq. 1. They represent slopes for numerical variables and an additive effect for the factor roughness

sequently, we derived the path lengths by calculating the Euclidean distances between particle positions in consecutive images. The calculated centre of mass could vary slightly from image to image by one or two pixels without any particle movement. Therefore, we ignored any trajectory of less than 3 pixels.

From every first image of the experiment, we estimated the sizes of the particles by segmenting the images at the grey value 200 (i.e. any pixel with a grey value equal to or larger than 200 was classified as particle). Based on these sizes in pixels, we calculated particle characteristics 'equivalent diameter' (i.e. the diameter that a completely spherical particle with the same area visible in the image would have) and 'eccentricity' which shows whether a particle resembles a sphere or an ellipse. Eccentricity is defined as the ratio between the focal points of an ellipse to its major axis. To calculate eccentricity, the particle's shape is approximated as an ellipse with the same secondorder moments (covariance) as its visible area. Both characteristics were calculated with the Python module 'measure' from the image processing library scikit-image (https://scikit-image.org/).

\section{Statistical analysis}

We estimate the influence, i.e. effect sizes and confidence intervals, of the experimental parameters 'roughness', 'inclination,' 'irrigation' rate and 'equivalent diameter' on the length of paths that the particles moved in the experimental runs. We follow the advice of the American Statistical Association and refrain from hypothesis testing $[49,50]$. Instead, we estimate the effect sizes and their confidence intervals using bootstrap re-sampling [51] in the following linear model:

$$
y=\operatorname{Int}+\beta_{\mathrm{ED}} \cdot \mathrm{ED}+\beta_{\mathrm{Inc}} \cdot \operatorname{Inc}+\beta_{\mathrm{Irr}} \cdot \operatorname{Irr}+\beta_{\mathrm{Rough}} \cdot \operatorname{Rough}+\varepsilon
$$

where $y$ is the path length in $\mathrm{mm}$, Int the intercept in $\mathrm{mm}$, ED the equivalent diameter in $\mu \mathrm{m}$, Inc the inclination in degrees, Irr the irrigation rate in $\mathrm{L} \mathrm{h}^{-1}$, Rough the roughness (a factor, coarse being the reference level), $\beta_{i}$ are the model parameters or effects (a slope for numerical variables and an additive effect for factors) and $\varepsilon$ are the residuals of the model. The intercept is the expected value of the path length if all other factors are zero. This is not
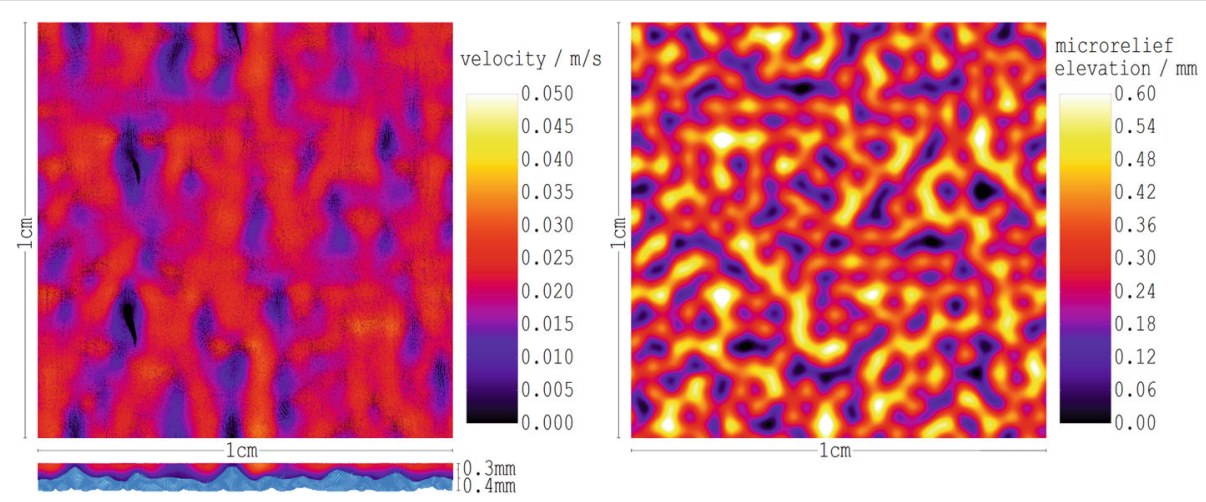

Fig. 5 Velocity profile on a $1 \mathrm{~cm} \times 1 \mathrm{~cm}$ section of the fine-grained microrelief illustrated with colored passive tracer particles (left). The $0.3 \mathrm{~mm}$ average water film thickness leads to a flow rate of $7.27 \mathrm{~L} \mathrm{~h}^{-1}$. The flow direction is top to bottom. The flow pattern is stationary and forms streams of increased velocity around teardrop shaped regions of decreased velocity behind bumps in the microrelief. At the bottom left we show a side view of the microrelief and water layer above. At the right we show a heatmap of the microrelief elevation 
meaningful for our setting because this would extrapolate the model beyond the measured ranges of the experimental parameters. To have a meaningful intercept, we subtracted the mean from the irrigation, inclination and the equivalent diameter, i.e. we centered all experimental parameters around their respective mean values. Therefore, the intercept now equals the travelled path length in $\mathrm{mm}$ for a mean-sized particle $(1817 \mu \mathrm{m})$ irrigated by the mean irrigation rate $\left(7.5 \mathrm{~L} \mathrm{~h}^{-1}\right)$, on a mean-inclined $\left(8.7^{\circ}\right)$ coarse surface. This data normalization does not affect the estimation of the effects $\beta_{i}$, but of the intercept only. We note that our model aims at a phenomenological description of particle transport within a certain range of parameters around their mean values rather than a fully detailed physical prediction.

We estimated the confidence intervals by bootstrapping the data (stratified by roughness) 10000 times and re-calculated the model on theses bootstrap samples [52]. Different methods to estimate confidence intervals from bootstraping exist and we compared three of them, namely the percentile, student- $t$ and bca (biascorrected and accelerated) methods, all implemented in the R package rsample [53]. All calculations were done in $R$ [47] using the package collection tidymodels [54], V.0.1.0.

\section{Simulation of the water flow}

To better understand the water flow on the sediment surfaces, we qualitatively examined the flow patterns in a simulation on a microscopic level of detail. We assumed a free surface flow totally wetting an inclined plate with roughness at the scale of the grains of sand used in preparation of the fine surface. The liquid layer was very thin, about $0.1-0.3 \mathrm{~mm}$ thickness.

To model the flow, we used the Volume-of-Fluid [55-57] lattice Boltzmann method [58, 59] implemented in the software FluidX3D $[55,60,61]$. We simulated an area of $10 \mathrm{~mm} \times 10 \mathrm{~mm}$ at $2.5^{\circ}$ inclination. The boundaries in the lateral directions were periodic. We used the parameters of water at $20{ }^{\circ} \mathrm{C}$ (kinematic shear viscosity $v=1.004 \cdot 10^{-6} \mathrm{~m}^{2} \mathrm{~s}^{-1}$, density $\rho=998.21 \mathrm{~kg} \mathrm{~m}^{-3}$, surface tension $\sigma=72.75 \cdot 10^{-3} \mathrm{~kg} \mathrm{~s}^{-2}$ ) [62]. We generated the microrelief of the surface caused by its roughness with a 2D periodic Perlin noise [63]. The grain size for the Perlin noise was set to approximately $0.4 \mathrm{~mm}$ diameter and the thickness of the water film to $0.3 \mathrm{~mm}$ on average. This resulted in a simulated flow rate (scaled up to the entire $400 \mathrm{~mm}$ width of the plate in the experiment) of $7.27 \mathrm{~L} \mathrm{~h}^{-1}$ which is in line with experimental parameter of $7.2 \mathrm{~L} \mathrm{~h}^{-1}$.

Additionally, to visualize the flow patterns, we irrigated the surfaces without any PMMA particles with a methanol solution stained with Nile Red, a fluorescent dye [64, 65]. Using Nile Red allowed us to capture the flow patterns with the same LED lights and camera setup and filter as in the experimental runs with particles. We are aware that methanol has different wetting properties than water. Therefore, we use the videos for visualisation purposes only. We recorded the initial wetting process as well as the flow patterns once stationary flow set in. The coarse surface was irrigated at an inclination of $7.5^{\circ}$ with an irrigation rate of $7.2 \mathrm{~L} \mathrm{~h}^{-1}$ and the fine surface at an inclination of $2.5^{\circ}$ with an irrigation rate of $7.2 \mathrm{~L} \mathrm{~h}^{-1}$. The videos are provided in the Additional files 2 and 3 .

\section{Results and discussion}

\section{Tracking microplastics under laboratory conditions}

Our experiments allowed for a high-resolution tracking of microplastic particles under different inclination, surface roughness and irrigation scenarios. Next to their respective trajectories, the image analysis provided information about the characteristics of the particles in the experimental runs. The equivalent diameter of PMMA particles ranged between 453 and $3387 \mu \mathrm{m}$ (Supplementary Figure S3). They differed from spheres with eccentricities varying between 0.1 and 0.9 . An eccentricity of 0 corresponds to a perfect circle, a value of 1 indicates an "extreme ellipse", a parabola. Thus, the larger the value the less circular the PMMA particle. The distribution of both characteristics were comparable between different roughnesses and irrigation rates and shows that the particles in the experiments were comparable (Supplementary Figure S3). Therefore, no differences between the treatments as a consequence of a systematically varying particle morphology are to be expected.

576 out of 1081 particles remained motionless and the other 505 recorded particle trajectories were mostly short (Fig. 3). Most of the recording time, particles did not move at all. However, from time to time they travelled a larger distance, often very quickly. Thus, the overall path lengths and the particle's maximum path length per second correlated (Spearman correlation coefficient of 0.9). The Supplementary Figure S5 shows this relationship. The maximum overall recorded path length equalled $136 \mathrm{~mm}$ (fine surface, inclination $10^{\circ}$, irrigation rate $10.44 \mathrm{~L} \mathrm{~h}^{-1}$ ) and the maximum recorded path length in one second $135 \mathrm{~mm}$ (fine surface, inclination $2.5^{\circ}$, irrigation rate 7.2 $\left.\mathrm{L} \mathrm{h}^{-1}\right)$.

\section{Influence of experimental parameters on path lengths}

The observed path lengths increased with increasing irrigation rate and were larger on the fine than on the coarse surface (Fig. 4). However, the dot plots show that the most frequent paths were small or zero and that only few particles traveled far.

The estimated parameters of the linear model support this observation (Table 1). Confidence intervals estimated with different bootstrap methods were comparable 
(Supplementary Figure S6) and we report the student- $t$ intervals only. All confidence intervals excluded zero. With increasing inclination and increasing equivalent diameter of the microplastic particles, the path length decreased. Indeed, for a constant flow rate, the water film thickness is approximately proportional to the $(\sin (\alpha))^{-1 / 3}$, with $\alpha$ being the inclination [66][p.183 eq.(4.2.13)] and the larger the film thickness the larger the flow rate (see also "Water flow" section). The effects of inclination and equivalent diameter seem smaller compared to the irrigation rate or the roughness. However, the experimental parameters are measured on different scales and the absolute values of the effects depend on it. Thus, it is better to compare the effects relative to the measurement units of the parameters. If, for example, the inclination is increased from 5 to 10 degrees, the path length would decrease by $2.3 \mathrm{~mm}$. For an increase of irrigation rate from $4 \mathrm{~L} \mathrm{~h}^{-1}$ to $8 \mathrm{~L} \mathrm{~h}^{-1}$, the path length would increase by $3.6 \mathrm{~mm}$. By increasing the equivalent diameter of the microplastic particles from 1000 to $2000 \mu \mathrm{m}$, the

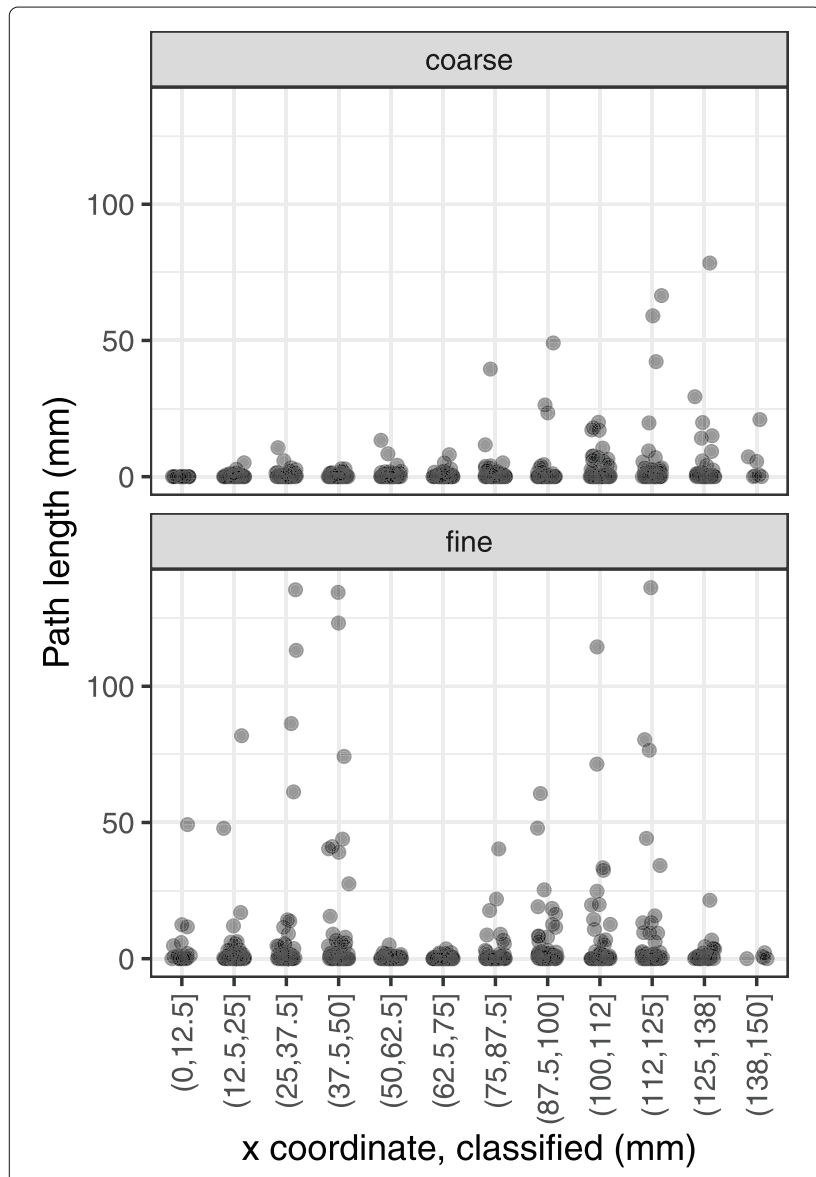

Fig. 6 Variation of path lengths with starting position of the particles on the $x$ axis. The $x$ coordinate of the starting point was split in classes of $12.5 \mathrm{~mm}$ length to better show the distribution of path lengths path length decreases by $3 \mathrm{~mm}$. And finally, on the fine surface, the particles travelled $3.33 \mathrm{~mm}$ farther which is in accordance with several observations from laboratory and environmental studies (e.g. in fluvial context and at valley slopes $[67,68]$ or on beaches $[69,70])$.

The overall goodness of fit of the model was low $\left(R_{\mathrm{adj}}^{2}=0.07\right)$ indicating that the assumption of the linear relationship between the experimental parameters and the path length is overly simplistic. However, because our intention is not to predict path lengths but to analyse the influence of experimental parameters (size effect and confidence intervals), the model is still useful.

\section{Water flow}

To better understand why most particles are barely advected whilst a few particles are moved across a long distance (Fig. 4), we qualitatively examined the flow pattern in a simulation. For the typical flow rates used in the experiment, the water film thickness is on the same scale as the grain size of the roughness-induced microrelief. The microrelief therefore greatly influences the flow pattern on a microscopic level. For the setup simulated here, where we target $7.2 \mathrm{~L} \mathrm{~h}^{-1}$ to match the experiment, we find that the film thickness is $0.3 \mathrm{~mm}$ on average.

For comparison, for the targeted irrigation rates of 4.8 and $10.4 \mathrm{~L} \mathrm{~h}^{-1}$, we determine the film thickness to $0.252 \mathrm{~mm}$ and $0.348 \mathrm{~mm}$ respectively. The resulting simulation flow rates are 4.87 and $10.97 \mathrm{~L} \mathrm{~h}^{-1}$. The flow rate is proportional to film thickness to the third power for a laminar flow over a plane and smooth surface [66][p.183 eq.(4.2.12)]. With increasing inclination, the film thickness decreases. If the film is thinner than the diameter of the microplastic particles, the water surface wetting the particles bulges up on them and pushes them down onto the microrelief, increasing friction and reducing mobility. In contrast, at large film thickness, microplastic particles may be entirely engulfed into the liquid and not touch the microrelief at all, reducing friction and increasing mobility. So although the flow velocity at the surface is faster (proportional to $(\sin (\alpha))^{1 / 3}$, with $\alpha$ being the inclination) at larger inclination, the friction between the particles and the plate is increased and particles are less mobile overall. The roughness changes the flow conditions and influences the flow rate. Therefore, this relationship holds only approximately.

Figure 5 shows the emerging flow pattern after $0.1 \mathrm{~s}$ of simulation, illustrated with colored passive tracer point-particles. These are passively advected points without influence on the flow itself. They are illustrated as colored pixels to show the magnitude of local velocity. 
Although the microrelief has no directionality in itself, the flow pattern shows distinctive channels with increased flow velocity on the scale of the microrelief, while in teardrop-shaped regions behind larger bumps of the microrelief, flow velocity is significantly reduced. This pattern is stationary. The asymmetry of the flow pattern indicates deviations from purely laminar flow. There are regions where particles will move down the plate with increased probability (fast flow channels) while in other regions the hydrodynamic force can never overcome friction.

At the low flow rates in the experiment, there are only little surface instabilities (non-stationary, bow-shaped, capillary waves) present. These mainly occur at higher flow rate and larger film thickness [71-77].

On top of the microrelief, the surfaces also possess a macrorelief. The latter emerged during construction of the plates when sand was glued to the chipboards. In regions where there is more glue, the sand layer may be thicker, and in other regions the sand layer may be thinner. This macrorelief also creates preferential flow channels (on a larger scale) where PMMA particles are transported particularly far. Figure 6 shows that on the coarse surface, particles starting on the right moved the farthest, while on the fine surface, preferential flow existed on both the left and right sides.

These macroscopic flow channels would be much more difficult to model in a simulation, since on the one hand both micro- and macrorelief would have to be simultaneously resolved on very different length scales and on the other hand the exact topography is of key importance because it directly affects experimental results. So to confirm those preferential macroscopic flow channels in the experimental setup, we used the recordings of methanol solution stained with Nile Red. Figure 7 confirms the preferential flow paths on the macro scale on the fine surface. The imaged part in the videos is slightly shifted compared to the imaged part in the experiments because the experimental setup had to be moved under the fume hood. The videos are provided in the Supporting Information Additional files 2 and 3.

In the video of the fine surface, we also observed some surface ripples, mainly along the preferential flow channels in the macrorelief. These may be caused by the locally increased flow rate, but could also be a result of the irrigation system perturbing the surface. Such surface ripples could temporarily increase the hydrodynamic force enough to unlock a microplastic particle from the microrelief and initiate the motion.

\section{Transport processes and environmental implications}

In our experiments, we observe that the transport of the PMMA particles is based on two different phenomena: transport-inhibiting interaction with the microrelief and transport-enhancing interaction with preferential flow channels of the macrorelief. In the first case flow patterns with areas of larger flow velocities and therefore larger hydrodynamic forces acting on the particles emerge due to the microrelief. The size of PMMA particles is at a similar scale as the microrelief sand grains and generally larger than the water film. This means that the particles on the one hand are able to interlock with the microrelief and are not transported, and on the other hand have constant friction if rolled down the plate by the flow, increasing the probability to lock into the microrelief again and get stuck after a short travel distance. Therefore, most particles did not move at all or travelled only a very short distance. Second, the macrorelief that overlays the microrelief provides macroscopic preferential flow channels for the particles. If a particle is located in or reaches a preferential flow channel, the travelled path lengths are large.

Under natural conditions, the fast flow pathways (both micro and macro) will potentially lead to increased erosion, carving channels into the soil surface [78, 79]. Gomez et al. [78], for example, observed a larger erosion on rougher surfaces and attributed this to "concentration of flow around roughness elements". This is comparable to the flow patterns around rough surface elements that we obtained in the simulation on the microscale and the observed preferential flow paths on the macroscale. This kind of 'flow concentration' could finally lead to rill erosion [80]. Additionally, under natural conditions, interrill erosion due to the detachment of particles by raindrop impact and transport to rills (i.e. preferential pathways), an interplay between overland flow, water infiltration and surface sealing could be important processes affecting particle transport. At least in the beginning, interrill erosion could be size-selective and small particles are eroded preferentially $[81,82]$. However, the exact distribution of eroded particles for a particular soil depends on different soil properties like particle size distribution or aggregate stability [83, 84]. The general dependence of soil erodibility is reflected in the well known Revised Universal Soil Loss Equation (RUSLE) [85]. It is still unclear whether erosion processes would affect microplastic particles in the same way as natural sediments because of the broad range of shapes, densities and sizes of microplastics $[7,8]$.

In the experiments and the simulation investigated here, erosion and infiltration are explicitly prevented. Furthermore it should be considered that the irregular PMMA particles of a mean particle length of $1215 \mu \mathrm{m}$ that we used to conduct our experiments are only one exemplary polymer and microplastics shape. Because microplastics comprise a large range of different particle sizes, densities, shapes and degradation stages, our results can reflect a small part of the reality only $[7,8]$. Particles of different shapes (e.g. spheres, fibres or films) and with different 

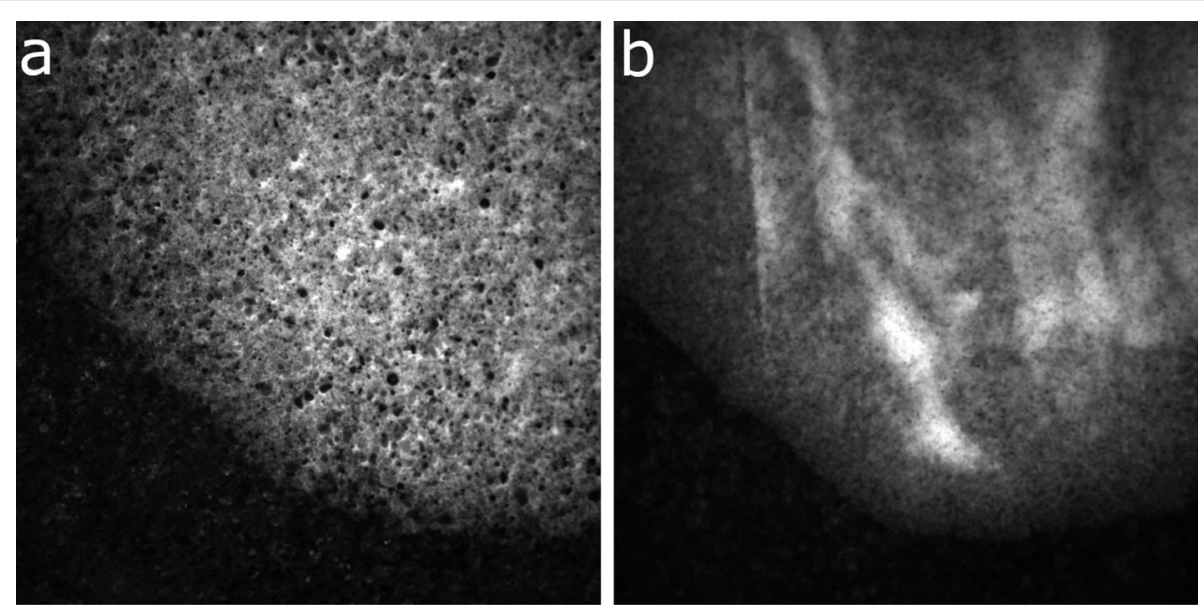

Fig. 7 Runoff stained with Nile red. a: coarse surface irrigated at $7.5^{\circ}$ inclination with $7.2 \mathrm{~L} \mathrm{~h}^{-1}$; b: fine surface irrigated at $2.5^{\circ}$ inclination with $7.2 \mathrm{~L} \mathrm{~h}^{-1}$. Preferential flow paths are visible on the fine surface

surface properties (e.g. due to degradation or biofilm) might interact differently with rough surfaces and be (re)mobilized and transported in a different way.

Nevertheless, the results of our laboratory setup contribute to understanding the horizontal transport of microplastics in nature. Sandy sediment and soil surfaces with sparse vegetation cover resemble to a certain extent to our surfaces. Comparable environments can be found for example in floodplains and on riverbanks [86, 87], on agricultural fields, in clearing areas, in freshly developed building sites and in ruderal habitats [88]. Because rivers are important pathways of microplastics $[13,89]$, the adjacent riparian zones and floodplains act as an interface between aquatic and terrestrial ecosystems. Therefore, they play an important role in the distribution of microplastics within the "plastic cycle", a term coined by Horton et al. [20] to emphasize the connectivity between environmental compartments. During runoff events caused by floods or precipitation excess, microplastics can be (re-)mobilised, transported and accumulated in these highly dynamic areas $[44,87,90]$.

The transport parameters of microplastics under natural conditions such as the flow velocities necessary to move the particles, the influence of land cover (e.g. vegetation) and sedimentation rates [90] are still not deciphered. While Nizzetto and colleagues [31] suggested that microplastics moved similarly to natural particles and organic matter, there is still a significant lack of data to understand the transport patterns of microplastics "across the compartments of hydrological catchments" [44]. Analysing transport patterns under laboratory conditions may facilitate future studies on microplastic distribution and mobility. For future research additional important factors, e.g. topography, hydrology, land cover and the exposure of microplastics to physical, biological and chemical processes should be considered [44] in order to improve the understanding of microplastic transport and the resulting contamination of the environment.

\section{Conclusions}

We developed a laboratory setup to reliably trace fluorescent microplastic particles in real-time during irrigation experiments. By limiting the experimental variables to irrigation rate, inclination and surface roughness the driving factors of the particle movement could be deciphered. Especially the roughness and the irrigation rate turned out to be important. In our experiments, we could show that the transport of the microplastics was inhibited by the interaction of the microplastics with the microrelief and enhanced by preferential flow channels of the macrorelief of the rough surfaces. The computer simulated flow patterns showed variable flow velocities on the scale of the microrelief and thus spatially variable hydrodynamic forces acting on the particles. Our laboratory results are a first step to gain a better understanding of the horizontal transport of microplastics on natural sediment and soil surfaces. However, microplastics are a diverse group of contaminants with varying shapes, densities and sizes. This continuity of properties, alteration of the microplastic particles' surface properties as a consequence of biological and chemical processes in the environment and additional factors influencing soil surface, e.g. topography or land cover, could not be taken into account here and need to be part of further research.

\footnotetext{
Abbreviations

PMMA: Polymethyl methacrylate; sCMOS: Scientific complementary metal-oxide-semiconductor; CCD: Charge-coupled device
} 


\section{Supplementary Information}

The online version contains supplementary material available at https://doi.org/10.1186/s43591-021-00010-2.

Additional file 1: A pdf file with Supplementary Figures referenced in the main text.

Additional file 2: A video of runoff stained with Nile Red on the coarse surface. The surface was inclined by 7.5 degrees and irrigated with $7.2 \mathrm{~L} \mathrm{~h}^{-1}$

Additional file 3: A video of runoff stained with Nile Red on the fine surface. The surface was inclined by 2.5 degrees and irrigated with $7.2 \mathrm{~L} \mathrm{~h}^{-1}$.

\section{Acknowledgements}

The authors thank Julia Horn for support in the laboratory and Florian Steininger for technical assistance.

\section{Authors' contributions}

$\mathrm{CB}, \mathrm{HL}$ and MK designed the study, $\mathrm{HL}$ supervised the experimental work, ML simulated the water flow, MK did the irrigation experiments, MGJL prepared and characterized the PMMA particles, CB wrote the code and analysed the image data, all authors discussed the results, $\mathrm{HL}, \mathrm{CB}$ and $\mathrm{ML}$ wrote the first draft of the manuscript. All authors read and approved the final manuscript.

\section{Funding}

This project was funded by the Deutsche Forschungsgemeinschaft (DFG, German Research Foundation), Project Number 391977956, SFB 1357 , subprojects B04 and B06. We further acknowledge support through the computational resources provided by the Bavarian Polymer Institute. Open Access funding enabled and organized by Projekt DEAL.

\section{Availability of data and materials}

The datasets and code used and/or analysed during the current study are published on Zenodo, https://doi.org/10.5281/zenodo.4911185.

\section{Declarations}

Competing interests

The authors declare that they have no competing interests.

\section{Author details}

${ }^{1}$ Ecosystem Research Group, Institute of Geography, Faculty of Mathematics and Natural Sciences, University of Cologne, Albertus Magnus Platz, 50923 Cologne, Germany. ${ }^{2}$ Biofluid Simulation and Modeling - Theorethische Physik VI, University of Bayreuth, 95448 Bayreuth, Germany. ${ }^{3}$ Animal Ecology I, BayCEER, University of Bayreuth, 95440 Bayreuth, Germany.

\section{Received: 4 March 2021 Accepted: 7 June 2021}

Published online: 02 July 2021

\section{References}

1. Geyer R, Jambeck JR, Law KL. Production, use, and fate of all plastics ever made. Sci Adv. 2017;3(7):1700782. https://doi.org/10.1126/sciadv. 1700782.

2. PlasticsEurope. Plastics - the Facts 2020. Technical report. 2020. https:// www.plasticseurope.org/en/resources/publications/4312-plastics-facts2020. Accessed 25 June 2021.

3. Thompson RC, Olsen Y, Mitchell RP, Davis A, Rowland SJ, John AWG, McGonigle D, Russell AE. Lost at Sea: Where Is All the Plastic? Science. 2004;304(5672):838. https://doi.org/10.1126/science.1094559.

4. Arthur C, Baker J, Bamford H. Proceedings of the International Research Workshop on the Occurrence, Effects and Fate of Microplastic Marine Debris. Sept 9-11, 2008. NOAA Technical Memorandum NOS-OR\&R-30. 2009.

5. Kataoka T, Nihei Y, Kudou K, Hinata H. Assessment of the sources and inflow processes of microplastics in the river environments of Japan. Environ Pollut. 2019;244:958-65. https://doi.org/10.1016/j.envpol.2018.10. 111.

6. Meides N, Menzel T, Poetzschner B, Löder MGJ, Mansfeld U, Strohriegl $P$, Senker J. Reconstructing the Environmental Degradation of
Polystyrene by Accelerated Weathering. Environ Sci Technol. 2021;55(12): 7930-7938. https://doi.org/10.1021/acs.est.0c07718.

7. Rochman CM, Brookson C, Bikker J, Djuric N, Earn A, Bucci K, Athey S, Huntington A, Mcllwraith H, Munno K, Frond HD, Kolomijeca A, Erdle L, Grbic J, Bayoumi M, Borrelle SB, Wu T, Santoro S, Werbowski LM, ZhuX, Giles RK, Hamilton BM, Thaysen C, Kaura A, Klasios N, Ead L, Kim J, Sherlock C, Ho A, Hung C. Rethinking microplastics as a diverse contaminant suite. Environ Toxicol Chem. 2019;38(4):703-11. https://doi. org/10.1002/etc.4371.

8. Kooi M, Koelmans AA. Simplifying Microplastic via Continuous Probability Distributions for Size, Shape, and Density. Environ Sci Technol Lett. 2019;6(9):551-7. https://doi.org/10.1021/acs.estlett.9b00379.

9. Waller CL, Griffiths HJ, Waluda CM, Thorpe SE, Loaiza I, Moreno B, Pacherres CO, Hughes KA. Microplastics in the Antarctic marine system: An emerging area of research. 2017;598:220-7. https://doi.org/10.1016/j. scitotenv.2017.03.283

10. Horton AA, Walton A, Spurgeon DJ, Lahive E, Svendsen C. Microplastics in freshwater and terrestrial environments: Evaluating the current understanding to identify the knowledge gaps and future research priorities. Sci Total Environ. 2017;586:127-41. https://doi.org/10.1016/j. scitotenv.2017.01.190.

11. Bergmann M, Mützel S, Primpke S, Tekman MB, Trachsel J, Gerdts G. White and wonderful? Microplastics prevail in snow from the Alps to the Arctic. Sci Adv. 2019;5(8):1157. https://doi.org/10.1126/sciadv.aax1157.

12. Hurley RR, Nizzetto L. Fate and occurrence of micro(nano)plastics in soils: Knowledge gaps and possible risks. Curr Opin Environ Sci Health. 2018;1: 6-11. https://doi.org/10.1016/j.coesh.2017.10.006.

13. Rochman CM. Microplastics research-from sink to source. Science. 2018;360(6384):28-9. https://doi.org/10.1126/science.aar7734.

14. Rillig MC, Lehmann A. Microplastic in terrestrial ecosystems. Science. 2020;368(6498):1430-1. https://doi.org/10.1126/science.abb5979.

15. Löder MGJ, Gerdts G. Methodology Used for the Detection and Identification of Microplastics-A Critical Appraisal. In: Bergmann M, Gutow L, Klages M, editors. Marine Anthropogenic Litter. Cham: Springer International Publishing; 2015. p. 201-27.

16. Bläsing M, Amelung W. Plastics in soil: Analytical methods and possible sources. Sci Total Environ. 2018;612:422-35. https://doi.org/10.1016/j. scitotenv.2017.08.086.

17. Piehl S, Leibner A, Löder MGJ, Dris R, Bogner C, Laforsch C. Identification and quantification of macro- and microplastics on an agricultural farmland. Sci Rep. 2018;8(1):17950. https://doi.org/10.1038/ s41598-018-36172-y.

18. Möller JN, Löder MGJ, Laforsch C. Finding Microplastics in Soils: A Review of Analytical Methods. Environ Sci Technol. 2020;54(4):2078-90. https:// doi.org/10.1021/acs.est.9b04618.

19. Möller JN, Heisel I, Satzger A, Vizsolyi EC, Oster J, Agarwal S, Laforsch C, Löder MGJ. Tackling the Challenge of Extracting Microplastics from Soils: Protocol to Purify Soil Samples for Spectroscopic Analysis. Environ Toxicol Chem. 2021. https://doi.org/10.1002/etc.5024.

20. Horton AA, Dixon SJ. Microplastics: An introduction to environmental transport processes. Wiley Interdiscip Rev: Water. 2018;5(2):1268. https:// doi.org/10.1002/wat2.1268

21. Weithmann N, Möller JN, Löder MGJ, Piehl S, Laforsch C, Freitag R. Organic fertilizer as a vehicle for the entry of microplastic into the environment. Sci Adv. 2018;4(4):8060. https://doi.org/10.1126/sciadv. aap8060.

22. Allen $S$, Allen $D$, Phoenix VR, Le Roux G, Durántez Jiménez $P$ Simonneau A, Binet S, Galop D. Atmospheric transport and deposition of microplastics in a remote mountain catchment. Nat Geosci. 2019;12(5): 339-44. https://doi.org/10.1038/s41561-019-0335-5.

23. Laforsch C, Ramsperger AFRM, Mondellini S, Galloway TS. Microplastics: A Novel Suite of Environmental Contaminants but Present for Decades. In: Reichl F-X, Schwenk M, editors. Regulatory Toxicology. Berlin, Heidelberg: Springer; 2021. p. 1-26.

24. Rillig MC. Microplastic in Terrestrial Ecosystems and the Soil? Environ SC Technol. 2012;46(12):6453-4. https://doi.org/10.1021/es302011r.

25. Van Cauwenberghe L, Devriese L, Galgani F, Robbens J, Janssen CR. Microplastics in sediments: A review of techniques, occurrence and effects. Mar Environ Res. 2015;111:5-17. https://doi.org/10.1016/j. marenvres.2015.06.007. 
26. Waldschläger K, Schüttrumpf H. Infiltration Behavior of Microplastic Particles with Different Densities, Sizes, and Shapes-From Glass Spheres to Natural Sediments. Environ Sci Technol. 2020;54(15):9366-73. https:// doi.org/10.1021/acs.est.0c01722.

27. Yu M, van der Ploeg M, Lwanga EH, Yang X, Zhang S, Ma X, Ritsema CJ, Geissen $V$. Leaching of microplastics by preferential flow in earthworm (Lumbricus terrestris) burrows. Environ Chem. 2019;16(1):31. https://doi. org/10.1071/EN18161.

28. Keller AS, Jimenez-Martinez J, Mitrano DM. Transport of Nano- and Microplastic through Unsaturated Porous Media from Sewage Sludge Application. Environ Sci Technol. 2020;54(2):911-20. https://doi.org/10. 1021/acs.est.9b06483.

29. Huerta Lwanga $E$, Gertsen $H$, Gooren $H$, Peters $P$, Salánki T, van der Ploeg M, Besseling E, Koelmans AA, Geissen V. Incorporation of microplastics from litter into burrows of Lumbricus terrestris. Environ Pollut. 2017;220:523-31. https://doi.org/10.1016/j.envpol.2016.09.096.

30. Rillig MC, Ziersch L, Hempel S. Microplastic transport in soil by earthworms. Sci Rep. 2017;7(1):1362. https://doi.org/10.1038/s41598-01701594-7.

31. Nizzetto L, Bussi G, Futter MN, Butterfield D, Whitehead PG. A theoretical assessment of microplastic transport in river catchments and their retention by soils and river sediments. Environ Sci Pollut Impacts. 2016;18(8):1050-9. https://doi.org/10.1039/C6EM00206D.

32. Black KS, Athey S, Wilson P, Evans D. The use of particle tracking in sediment transport studies: A review. Geol Soc Lond Spec Publ. 2007;274(1):73-91. https://doi.org/10.1144/GSL.SP.2007.274.01.09.

33. White TE. Status of measurement techniques for coastal sediment transport. Coast Eng. 1998;35(1-2):17-45.

34. Liedermann M, Tritthart M, Habersack H. Particle path characteristics at the large gravel-bed river Danube: Results from a tracer study and numerical modelling. Earth Surf Process Landforms. 2013;38:512-522. https://doi.org/10.1002/esp.3338.

35. McComb P, Black K. Detailed Observations of Littoral Transport Using Artificial Sediment Tracer, in a High-Energy, Rocky Reef and Iron Sand Environment. J Coast Res. 2005;212:358-73. https://doi.org/10.2112/03574.1.

36. Vila-Concejo A, Ferreira Ó, Ciavola P, Matias A, Dias JMA. Tracer studies on the updrift margin of a complex inlet system. Mar Geol. 2004;208(1): 43-72. https://doi.org/10.1016/j.margeo.2004.04.020.

37. Katsuragi $\mathrm{H}$. Length and time scales of a liquid drop impact and penetration into a granular layer. J Fluid Mech. 2011;675:552-73. https:// doi.org/10.1017/jfm.2011.31.

38. Long EJ, Hargrave GK, Cooper JR, Kitchener BGB, Parsons AJ, Hewett CJM, Wainwright J. Experimental investigation into the impact of a liquid droplet onto a granular bed using three-dimensional, time-resolved, particle tracking. Phys Rev E. 2014;89(3):032201. https://doi.org/10.1103/ PhysRevE.89.032201.

39. Hardy RA, Pates JM, Quinton JN, Coogan MP. A novel fluorescent tracer for real-time tracing of clay transport over soil surfaces. CATENA. 2016;141:39-45. https://doi.org/10.1016/j.catena.2016.02.011.

40. Hardy RA, James MR, Pates JM, Quinton JN. Using real time particle tracking to understand soil particle movements during rainfall events. CATENA. 2017;150:32-8. https://doi.org/10.1016/j.catena.2016.11.005.

41. Teleki PG. Fluorescent sand tracers. J Sed Res. 1966:36(2):468-85. https:// doi.org/10.1306/74D714EC-2B21-11D7-8648000102C1865D.

42. Rinnan R, Rinnan $\AA$. Application of near infrared reflectance (NIR) and fluorescence spectroscopy to analysis of microbiological and chemical properties of arctic soil. Soil Biol Biochem. 2007;39(7):1664-73. https://doi. org/10.1016/j.soilbio.2007.01.022.

43. Hardy RA, Quinton JN, James MR, Fiener P, Pates JM. High precision tracing of soil and sediment movement using fluorescent tracers at hillslope scale. Earth Surf Process Landf. 2019;44(5):1091-9. https://doi. org/10.1002/esp.4557.

44. Windsor FM, Durance I, Horton AA, Thompson RC, Tyler CR, Ormerod SJ. A catchment-scale perspective of plastic pollution. Glob Chang Biol. 2019;25(4):1207-21. https://doi.org/10.1111/gcb.14572.

45. Schneider CA, Rasband WS, Eliceiri KW. NIH Image to ImageJ: 25 years of image analysis. Nat Methods. 2012;9(7):671-5. https://doi.org/10.1038/ nmeth.2089.
46. Saurabh S, Maji S, Bruchez MP. Evaluation of sCMOS cameras for detection and localization of single Cy5 molecules. Opt Express. 2012;20(7):7338-49. https://doi.org/10.1364/OE.20.007338.

47. R Core Team. R: A Language and Environment for Statistical Computing Vienna, Austria: R Foundation for Statistical Computing; 2020. https:// www.R-project.org/.

48. Allan DB, Caswell T, Keim NC, van der Wel CM. soft-matter/trackpy: Trackpy v0.4.2 (Version v0.4.2). Zenodo. 2019. http://doi.org/10.5281/ zenodo.3492186.

49. Wasserstein $\mathrm{RL}$, Lazar NA. The ASA Statement on p-values: Context, process, and purpose. Am Stat. 2016;70(2):129-33. https://doi.org/10. 1080/00031305.2016.1154108.

50. Wasserstein RL, Schirm AL, Lazar NA. Moving to a World Beyond "p < 0.05". Am Stat. 2019;73(sup1):1-19. https://doi.org/10.1080/00031305. 2019.1583913.

51. Efron B, Tibshirani RJ. An Introduction to the Bootstrap: CRC press; 1994.

52. Davison AC, Hinkley DV. Bootstrap Methods and Their Application, Cambridge Series in Statistical and Probabilistic Mathematics. Cambridge: Cambridge University Press; 1997. https://doi.org/10.1017/ CBO9780511802843.

53. Kuhn M, Chow F, Wickham H. Rsample: General Resampling Infrastructure. 2020. R package version 0.0.7. https://CRAN.R-project.org/ package=rsample.

54. Kuhn M, Wickham H. Tidymodels: a Collection of Packages for Modeling and Machine Learning Using Tidyverse Principles. 2020. https://www. tidymodels.org.

55. Lehmann M. High Performance Free Surface LBM on GPUs. 2019. Master thesis, Biofluid Simulation and Modeling - Theorethische Physik VI, University of Bayreuth. Germany. https://doi.org/10.15495/ EPub_UBT_00005400.

56. Körner C, Thies M, Hofmann T, Thürey N, Rüde U. Lattice Boltzmann model for free surface flow for modeling foaming. J Stat Phys. 2005;121(1-2):179-96.

57. Janßen C, Krafczyk M. Free surface flow simulations on GPGPUs using the LBM. Comput Math Appl. 2011;61(12):3549-63.

58. Krüger T, Kusumaatmaja H, Kuzmin A, Shardt O, Silva G, Viggen EM. The lattice Boltzmann method. Springer Int Publ. 2017;10:978-3.

59. Chapman S, Cowling TG, Burnett D. The Mathematical Theory of Non-uniform Gases: an Account of the Kinetic Theory of Viscosity, Thermal Conduction and Diffusion in Gases. Cambridge, United Kingdom: Cambridge university press; 1990.

60. HäusI F. MPI-based multi-GPU extension of the Lattice Boltzmann Method. 2019. Bachelor thesis, Biofluid Simulation and Modeling Theorethische Physik VI, University of Bayreuth. Germany.

61. Lehmann M, Gekle S. Analytic solution to the piecewise linear interface construction problem and its application in curvature calculation for volume-of-fluid simulation codes. arXiv preprint arXiv:2006.12838. 2020. https://arxiv.org/abs/2006.12838.

62. Edge E. Water - Density Viscosity Specific Weight. https://www. engineersedge.com/physics/water_density_viscosity_specific_weight_ 13146.htm. Accessed 12 Feb 2021

63. Gustavson S. Perlin Noise. https://github.com/stegu/perlin-noise Accessed 12 Feb 2021.

64. Erni-Cassola G, Gibson MI, Thompson RC, Christie-Oleza JA. Lost, but Found with Nile Red: A Novel Method for Detecting and Quantifying Small Microplastics (1 mm to $20 \mu \mathrm{m}$ ) in Environmental Samples. Environ Sci Technol. 2017;51(23):13641-8. https://doi.org/10.1021/acs.est.7b04512.

65. Konde S, Ornik J, Prume JA, Taiber J, Koch M. Exploring the potential of photoluminescence spectroscopy in combination with Nile Red staining for microplastic detection. Mar Pollut Bull. 2020;159:111475. https://doi. org/10.1016/j.marpolbul.2020.111475.

66. Batchelor CK, Batchelor G. An Introduction to Fluid Dynamics. Cambridge: Cambridge university press; 2000

67. Hassan MA, Reid I. The influence of microform bed roughness elements on flow and sediment transport in gravel bed rivers. Earth Surf Process Landf. 1990;15(8):739-50. https://doi.org/10.1002/esp.3290150807.

68. Chaplot V, Poesen J. Sediment, soil organic carbon and runoff delivery at various spatial scales. CATENA. 2012;88(1):46-56. https://doi.org/10.1016/ j.catena.2011.09.004. 
69. Bauer BO, Davidson-Arnott RGD, Hesp PA, Namikas SL, Ollerhead J, Walker IJ. Aeolian sediment transport on a beach: Surface moisture, wind fetch, and mean transport. Geomorphology. 2009;105(1-2):106-16,. https://doi.org/10.1016/j.geomorph.2008.02.016.

70. Otvos EG. Rain-Induced Beach Processes; Landforms of Ground Water Sapping and Surface Runoff. J Coast Res. 1999;15(4):16.

71. Liu J, Paul JD, Gollub JP. Measurements of the primary instabilities of film flows. J Fluid Mech. 1993;250:69-101.

72. Ramaswamy B, Chippada S, Joo S. A full-scale numerical study of interfacial instabilities in thin-film flows. J Fluid Mech. 1996;325:163-94.

73. Gjevik B. Occurrence of finite-amplitude surface waves on falling liquid films. Phys Fluids. 1970;13(8):1918-25.

74. Lu C, Jiang S-Y, Duan R-Q. Wave characteristics of falling film on inclination plate at moderate reynolds number. Sci Technol Nucl Installations. 2016;2016:7. Article ID 6586097. https://doi.org/10.1155/ 2016/6586097.

75. Roy R, Jain S. A study of thin water film flow down an inclined plate without and with countercurrent air flow. Exp Fluids. 1989;7(5):318-28.

76. Yu Y, Cheng X. Experimental study of water film flow on large vertical and inclined flat plate. Prog Nucl Energy. 2014;77:176-86.

77. Ramadurgam S, Chakravarthy R, Tomar G, Govindarajan R. Stability of developing film flow down an inclined surface. Phys Fluids. 2012;24(10): 102109.

78. Gómez J, Nearing M. Runoff and sediment losses from rough and smooth soil surfaces in a laboratory experiment. CATENA. 2005;59(3):253-66.

79. Zhao L, Huang C, Wu F. Effect of microrelief on water erosion and their changes during rainfall. Earth Surf Process Landf. 2016;41(5):579-86. https://doi.org/10.1002/esp.3844.

80. Gilley JE. EROSION | Water-Induced. In: Hillel D, editor. Encyclopedia of Soils in the Environment. Oxford: Elsevier; 2005. p. 463-9. https://doi.org/ 10.1016/B0-12-348530-4/00262-9.

81. Miller WP, Baharuddin MK. Particle Size of Interrill-eroded Sediments from Highly Weathered Soils. Soil Sci Soc Am J. 1987;51(6):1610-5. https://doi. org/10.2136/sssaj1987.03615995005100060037x.

82. Asadi H, Moussavi A, Ghadiri H, Rose CW. Flow-driven soil erosion processes and the size selectivity of sediment. J Hydrol. 2011;406(1): 73-81. https://doi.org/10.1016/j.jhydrol.2011.06.010.

83. Wang L, Shi ZH. Size Selectivity of Eroded Sediment Associated with Soil Texture on Steep Slopes. Soil Sci Soc Am J. 2015;79:917-929. https://doi. org/10.2136/sssaj2014.10.0415.

84. Asadi $\mathrm{H}$, Ghadiri $\mathrm{H}$, Rose $\mathrm{CW}$, Rouhipour $\mathrm{H}$. Interrill soil erosion processes and their interaction on low slopes. Earth Surf Process Landf. 2007;32(5): 711-24. https://doi.org/10.1002/esp.1426.

85. Renard KG. Predicting Soil Erosion by Water: A Guide to Conservation Planning with the Revised Universal Soil Loss Equation (RUSLE): U.S. Department of Agriculture, Agricultural Research Service Agriculture Hadnbook Number 703; 1997.

86. Scheurer M, Bigalke M. Microplastics in Swiss Floodplain Soils. Environ Sci Technol. 2018;52(6):3591-8. https://doi.org/10.1021/acs.est.7b06003.

87. Weber CJ, Opp C. Spatial patterns of mesoplastics and coarse microplastics in floodplain soils as resulting from land use and fluvial processes. Environ Pollut. 2020;267:115390. https://doi.org/10.1016/j. envpol.2020.115390.

88. Fulazzaky MA, Khamidun MH, Yusof B. Sediment traps from synthetic construction site stormwater runoff by grassed filter strip. J Hydrol. 2013;502:53-61. https://doi.org/10.1016/j.jhydrol.2013.08.019.

89. Lebreton LCM, van der Zwet J, Damsteeg J-W, Slat B, Andrady A, Reisser J. River plastic emissions to the world's oceans. Nat Commun. 2017;8(1):15611. https://doi.org/10.1038/ncomms15611.

90. Lechthaler S, Esser V, Schüttrumpf H, Stauch G. Why analysing microplastics in floodplains matters: Application in a sedimentary context. Environ Sci Pollut Impacts. 2021;23(1):117-31. https://doi.org/10. 1039/DOEM00431F.

\section{Publisher's Note}

Springer Nature remains neutral with regard to jurisdictional claims in published maps and institutional affiliations.

\section{Submit your manuscript to a SpringerOpen ${ }^{\circ}$ journal and benefit from:}

- Convenient online submission

- Rigorous peer review

- Open access: articles freely available online

- High visibility within the field

- Retaining the copyright to your article

Submit your next manuscript at $>$ springeropen.com 